\title{
Evaluation of hyperelastic material properties based on instrumented indentation
}

\author{
V. I. Zafiropoulou \& A. E. Giannakopoulos \\ Laboratory of Strength of Materials and Micromechanics, \\ Department of Civil Engineering, University of Thessaly, Greece
}

\begin{abstract}
It is well known that instrumented indentation tests are useful tools in probing mechanical properties of materials such as metals and ceramics. Instrumented indentation of hyperelastic materials such as rubbers, bio-materials, tissues etc. has not been examined in depth, especially the inverse problem of material characterization from instrumented indentation response. The difficulty of the inverse problem for such materials is that the unknown property is a function, the elastic energy density function. There are several such functions and each function is often characterized by more than one parameter. If the maximum indentation depth is low, we have shown that instrumented indentation of initially unstretched hyperelastic materials can only resolve a combination of the material parameters. If the maximum indentation depth is high, the indentation can provide independent material properties, however not in a unique way. Moreover, high indentation loads could lead to surface puncturing and so blur the test results. In this work, we show that we can use spherical indentation of a substrate at different but known prestretch levels to obtain the involved material properties of the energy density function. The present methodology can also incorporate a limit energy failure criterion and instrumented indentation can incorporate this behavior which we may call indentation strength.

Keywords: instrumented indentation, hyperelasticity, prestretching, property extraction.
\end{abstract}

\section{Introduction}

In recent years, instrumented indentation has been used to estimate residual stresses and residual strains of elastoplastic materials. Important analysis has 
been conducted for equal-biaxial stress fields in the context of sharp indentation tests (see for example Bolshakov et al. [1], Suresh and Giannakopoulos [2]) and for general biaxial stress fields (see for example Giannakopoulos [3], Lee and Kwon [4], Bocciarelli and Maier [5], Larsson and Blanchard [6]). Important studies were also conducted for the influence of residual stresses to spherical indentation (see for example Swadener et al. [7] and Huber and Heerens [8]). We should point out that this prior work is focused on how to obtain residual stresses and strains from instrumented indentation. The present work suggests a reverse methodology: obtaining material properties with the help of residual strains. Although we will concentrate mainly on hyperelastic materials, the present ideas could be useful for the elastoplastic materials, breaking the often encountered "lack of uniqueness" of elastoplastic material constants (see for example Chen et al. [9]. Non linear finite element analysis was applied by Chang and Sun [10] to (frictionless) spherical indentation of an Ogden type hyperelastic half-space. The general form of the Ogden's strain energy function is:

$$
W\left(\lambda_{1}, \lambda_{2}, \lambda_{3}\right)=\sum_{k=1}^{m} \mu_{k}\left(\lambda_{1}^{a_{k}}+\lambda_{2}^{a_{k}}+\lambda_{3}^{a_{k}}-3\right) / a_{k}
$$

where $\lambda_{i}(i=1,2,3)$ define the principal values of the stretch tensor and $\mu_{k}$, $a_{k}$ are material constants. In the case of infinitesimal strains, the Ogden material behaves as an incompressible linear material with shear modulus $G$ (and elastic modulus $E$ )

$$
G=\frac{1}{2} \sum_{k=1}^{m} \mu_{k} a_{k}=\frac{E}{3} .
$$

The neo-Hookean material model is a special case of one-term Ogden $\operatorname{model}\left(m=1, a_{1}=2\right)$. The Mooney-Rivlin material is a two-term Ogden $\operatorname{model}\left(m=2, a_{k}= \pm 2, \mu_{k}= \pm C_{k}, k=1,2\right)$.

Chang and Sun [10] found that for a ratio of indentation depth/ball radius ( $h / R$ ) up to 0.29 , the load-indentation $(P-h)$ depth relation is approximated well by the classic Hertz solution:

$$
h=\left(\frac{9}{16} \frac{P^{2}}{E^{2} R}\right)^{1 / 3} .
$$

This $P-h$ result was found to be independent of other material parameters. Similar results have been found for the case of a flat punch and the cone punch for an extensive variety of strain energy functions (Giannakopoulos and Panagiotopoulos [11], Zisis et al. [12]). It is of interest to note that this lack of uniqueness of the $P-h$ curve has been also observed in the analysis of elastoplastic indentation by sharp indentors. In a most recent and extensive work on this subject, Chen et al. [9] came up with a whole class of elastoplastic materials that show the same $P-h$ curves. 
Spherical indentation of soft matter, beyond the Hertzian regime has been investigated numerically using hyperelastic models by Lin and Chen [13]. They found that Hertz solution applies for $h / R<0.2$, with the relation between the contact radius $a$, the ball radius $R$ and the indentation depth $h$ as

$$
h=a^{2} / R \text {. }
$$

For $h / R>0.2$, their results do not follow Hertz solution.

Green et al. [14] have investigated the flat punch problem of an initially isotropic hyperelastic substance under equal-biaxial finite deformation. Woo and Shield [15] investigated the semi-infinite media in biaxial extension with a superposed force normal to the boundary. Equibiaxially stressed neo-Hookean half-space indented by a punch of arbitrary axisymmetric profile has been investigated by Dhaliwal et al. [16] and for general hyperelastic (isotropic) bodies by Beatty and Usmani [17]. Karduna et al. [18] performed finite element analysis of a Mooney-Rivlin type of rubber under equal-biaxial stretching and confirmed the theoretical predictions of Humphrey et al. [19].

Using the incremental theory of Biot [20] and the potential theory, the results can be extended for the indentation by any smooth axisymmetric indenter. The $h-a$ relation is given by the linear elastic solution and the $P-h$ relation admits an overall correction that depends on the amount of the initial biaxial stretch $\lambda_{r}$. For a neo-Hookean material, the finite tensile stretching makes it harder to deform under indentation.

It is clear that extracting hyperelastic material properties from indentation tests is very complex. It fails to provide reliable and unique results, unless for high indentation depths. However, high indentation depth can cause damage of the material, especially for soft tissues. In this work we will explore the indentation method in combination with prestretching, in order to extract hyperelastic material properties.

\section{Initially isotropic, incompressible hyperelastic materials under equal-biaxial stretching}

In this work, we will focus on initially isotropic, incompressible hypereleastic materials. These materials are described by an elastic energy density of the form

$$
W=W\left(I_{1}, I_{2}\right)
$$

with $\quad I_{1}=\lambda_{1}^{2}+\lambda_{2}^{2}+\lambda_{3}^{2}, \quad I_{2}=\lambda_{1}^{2} \lambda_{2}^{2}+\lambda_{2}^{2} \lambda_{3}^{2}+\lambda_{3}^{2} \lambda_{1}^{2} \quad$ and $\quad$ the incompressibility condition $\lambda_{1} \lambda_{2} \lambda_{3}=1 \quad\left(\lambda_{1}, \lambda_{2}, \lambda_{3}\right.$ are the principal stretches). We will further assume that an elastic substrate, as described above has as initial equal-biaxial stretching along the coordinate system $(x, y, z)$, as shown in fig. 1, with principal stretches in the radial direction $r, \lambda_{1}=\lambda_{2}=\lambda$ and in the normal direction $Z, \lambda_{3}=1 / \lambda^{2}$. Therefore, $I_{1}=2 \lambda^{2}+\lambda^{-4}$, 
$I_{2}=\lambda^{4}+2 \lambda^{-2}$. On such substrate, we impose a rigid spherical indentor of radius $\mathrm{R}$, that indents the surface with a normal load $P$. Green et al. [14] have solved the problem analytically, assuming that the deformation due to indentation is small. The result for the force-depth $(P-h)$ relation and the contact radius-depth $(a-h)$ relation for the case of frictionless and adhesionless contact are

$$
\begin{aligned}
h^{3 / 2} & =\frac{9}{16} \frac{P}{\times \mathrm{ER}} \\
a & =\sqrt{h R}
\end{aligned}
$$

where $\mathrm{E}$ is the initial elastic modulus for the uniaxial case. The above results are similar to the classic Hertzian case, except for the factor $x$ which is a function of $\lambda$ and of the material constants, and is given explicitly by the relation

$$
\begin{gathered}
x(\lambda)=\frac{\left(k_{1} b+a\right) \sqrt{k_{1}}\left(1+k_{2}\right)-\left(k_{2} b+a\right) \sqrt{k_{2}}\left(1+k_{1}\right)}{2\left(k_{1}-k_{2}\right)} \\
W_{i}=\partial W / \partial I_{i}, W_{i j}=W_{j i}=\partial^{2} W / \partial I_{j} \partial I_{i} \\
c=4 \lambda^{2}\left[\left(W_{1}+\lambda^{2} W_{2}\right)+\left(\lambda^{2}-\lambda^{-4}\right)\left(W_{11}+W_{22} \lambda^{2}\left(\lambda^{-4}+\lambda^{2}\right)\right)+W_{12}\left(\lambda^{-4}+2 \lambda^{2}\right)\right] \\
d=4 \lambda^{-4}\left[\left(W_{1}+\lambda^{2} W_{2}\right)+\left(\lambda^{-4}-\lambda^{2}\right)\left(W_{11}+2 W_{22} \lambda^{4}+3 W_{12} \lambda^{2}\right)\right] \\
d=4 \lambda^{-4}\left[\left(W_{1}+\lambda^{2} W_{2}\right)+\left(\lambda^{-4}-\lambda^{2}\right)\left(W_{11}+2 W_{22} \lambda^{4}+3 W_{12} \lambda^{2}\right)\right] \\
k_{1,2}=\frac{-(a+b-c-d) \pm \sqrt{(a+b-c-d)^{2}-4 a b}}{2 b}
\end{gathered}
$$

Clearly, for the unstretched case, $\lambda=1$ and $x(1)=1$.

Note also that other type of rigid axisymmetric punches (flat circular, cone, etc.) follow the classic solutions of Harding and Sneddon [21] with the substitutions: $\quad v \rightarrow 1 / 2 \quad$ (incompressibility), $\mathrm{E} \rightarrow x(\lambda) \mathrm{E} \quad$ (stretching influence). The indentation is assumed to be performed relatively fast, to avoid viscoelastic effects, e.g. Martinez-Martinez and De Hosson [22]. 


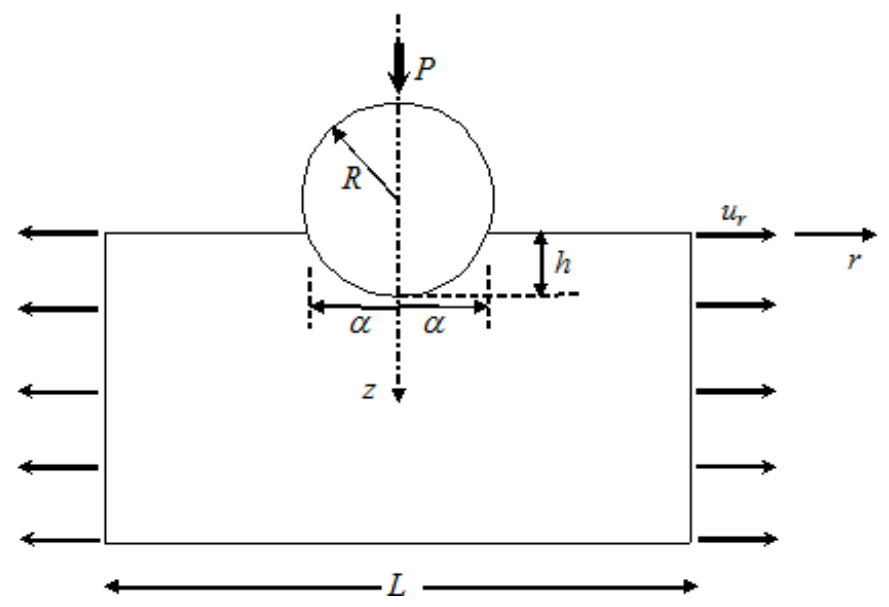

Figure 1: A spherical indenter is pushed by a force $P$ into a hyperelastic substrate up to a vertical displacement $h . \alpha$ is the contact radius and $R$ is the radius of the sphere, $\lambda=1+u_{r} / L$.

The case of the Mooney-Rivlin model is described by

$$
W=C_{1}\left(I_{1}-3\right)+C_{2}\left(I_{2}-3\right)
$$

where $C_{1}, C_{2}$ are material parameters and $\mathrm{E}=6\left(C_{1}+C_{2}\right)>0$. In this case

$$
x(\lambda)=\frac{\lambda^{9}+\lambda^{6}+3 \lambda^{2}-1}{2 \lambda^{4}\left(\lambda^{3}+1\right)}\left[\frac{C_{1}}{C_{1}+C_{2}}\left(1-\lambda^{2}\right)+\lambda^{2}\right] .
$$

For the particular case of a Neo-Hookean material $C_{2}=0, \mathrm{E}=6 C_{1}$. Note that eqn. (13) suggests that for $\lambda \geq 1, x(\lambda) \geq 1$ (and for $\lambda \leq 1, x(\lambda) \leq 1$ ) indicating a stiffening effect of the biaxial tension. Also $x(\lambda)$ is linearly increasing with respect to $C_{1} /\left(C_{1}+C_{2}\right)$, since $0 \leq C_{1} /\left(C_{1}+C_{2}\right) \leq 1$. From fig. 2 we can observe that we will need a prestretch $\lambda>1.5$ in order for $x$ to differ significantly from 1 . This is because as $C_{1} /\left(C_{1}+C_{2}\right) \rightarrow 1$, then $x(\lambda)$ tends to the Neo-Hookean result, (i.e. $C_{2} \rightarrow 0$, and in this case $x(\lambda)$ does not deviate much from 1 for $1<\lambda<1.5$.

At this point, we can make a very important observation. An instrumented indentation test of an unstretched Mooney-Rivlin surface $(\lambda=1)$ is likely to provide the value of $\mathrm{E}\left(=6\left(C_{1}+C_{2}\right)\right)$, but not the independent material 


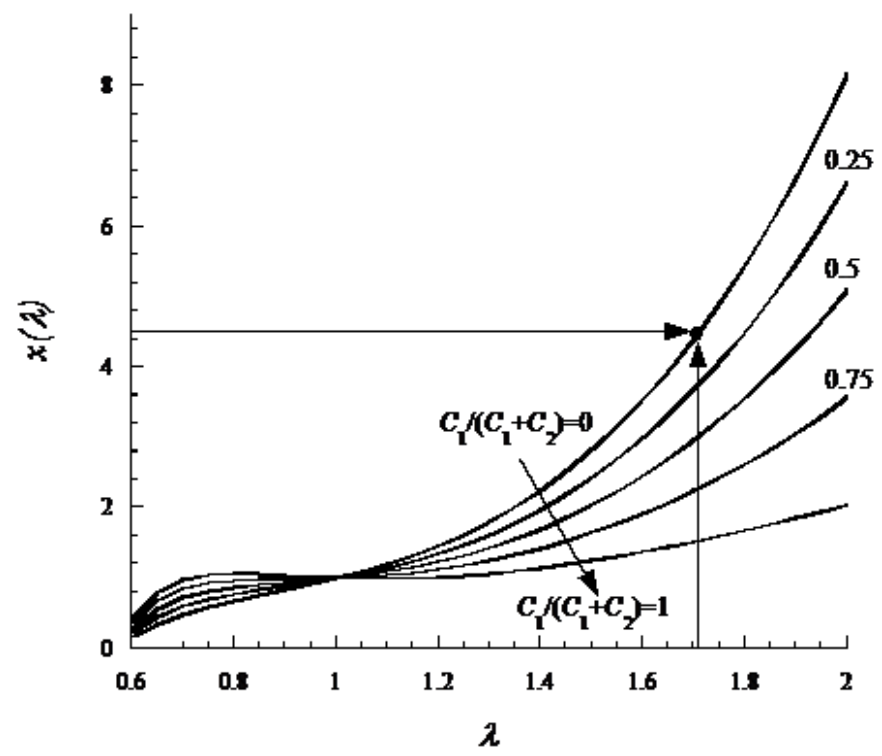

Figure 2: The variable $x(\lambda)$ for the Mooney-Rivlin material. We also show the proposed methodology, as applied to the Mooney-Rivlin material. Step 1: Perform instrumented indentation for $\lambda=1$ (unstretched substrate). Evaluate $E$ from $P-h$ experiments and so evaluate $C_{1}+C_{2}=E / 6$. Step 2: Perform instrumented indentation for $\lambda>1$ (known equal-biaxial stretch). Evaluate the $x(\lambda) E$ from $P-h$ experiment and therefore, $x(\lambda)$. From the analysis $\lambda$ and $x(\lambda)$ define a point corresponding to a district value of $C_{1} /\left(C_{1}+C_{2}\right)$. Step 3 : From $C_{1}+C_{2}$ and $C_{1} /\left(C_{1}+C_{2}\right)$ estimate uniquely $C_{1}$ and $C_{2}$ separately.

constants $C_{1}, C_{2}$ (unless for the Neo-Hookean material). If, however, we perform additional indentation tests with different levels of pre-stretch $\lambda$, then the values of $x(\lambda)$ could provide sufficient conditions to estimate all independent material constants. For the case of the Mooney-Rivlin model, one additional test with $\lambda>1$ is enough. The procedure for the model is shown in fig. 2, where knowledge of $\mathrm{E}$ and $\lambda$ can provide $C_{1}+C_{2}$ and $C_{1} /\left(C_{1}+C_{2}\right)$ respectively and so $C_{1}$ and $C_{2}$ can be found independently. The method can be applied likewise for other hyperelastic models. 
A further important note is that the proposed method requires $\lambda$ such that $x(\lambda)>0$. This implies that $\lambda$ cannot take values that make $x(\lambda) \leq 0$, because then the indentation becomes unstable under a compressive indentation load. For the case of the Mooney-Rivlin model, $\lambda^{9}+\lambda^{6}+3 \lambda^{2}-1>0$, which implies $\lambda>0.67$. This means that high compressive stretches $(\lambda<0.67)$ can be problematic for indentation and useless for the proposed procedure of material constant estimation via indentation. Obviously, if the material properties are known, then the initial stretch $\lambda$ (if any) can be estimated from indentation as follows. Instrumented indentation can provide $x \mathrm{E}$ and then, isolating the value of $x(\lambda)$, we can use the present analysis to obtain $\lambda$ (e.g. for a MooneyRivlin material solve eqn. (13) with respect to $\lambda$ ).

The present methodology was applied to a carbon filled rubber that follows the Yeoh's model (characterized by 3 constants, the second one being negative). By applying 4 different levels of prestretch, we were able to capture the three constants of the model quite reasonably (not shown here due to constrained space).

\section{Acknowledgements}

A.E.G. and V.I.F. are thankful to "ARISTEIA II" and "ARISTEIA I", respectively, Actions of the "Operational Programme Education and Life Long Learning”, co-founded by the European Social Fund (ESF) and National Resources.

\section{References}

[1] Bolshakov, A., Oliver, N.C. \& Pharr, G.M., Influences of stress on the measurement of mechanical properties using nano indentation. Part II. Finite element simulations, J. Mater. Res., 11, pp. 760-768, 1996.

[2] Suresh, S. \& Giannakopoulos, A.E., A new method for estimating residual stresses by instrumented sharp indentation. Acta Mater., 46, pp. 57555767, 1998.

[3] Giannakopoulos, A.E., The influence of initial elastic surface stresses on instrumented sharp indentation. J. Appl. Mech., 70, pp. 638-643, 2003.

[4] Lee, Y.H. \& Kwon, D., Estimation of biaxial surface stress by instrumented indentation with sharp indenters. Acta Mater., 56, pp. 1551563, 2004.

[5] Bocciarelli, M. \& Maier, G., Indentation and imprint mapping method for indentification of residual stresses, Computational Mater. Sci., 39, pp. 381-392, 2007.

[6] Larsson, P.-L. \& Blanchard, P., On the correlation between residual stresses and global indentation quantities: Numerical results for general biaxial stress fields. Materials and Design, 37, pp. 435-442, 2012. 
[7] Swadener, J.G., Taljat, B. \& Pharr, G.M., Measurement of residual stress by load and depth sensing indentation with spherical indenters. J. Mater. Res., 16, pp. 2091-2102, 2001.

[8] Huber, N. \& Heerens, J., On the effect of a general residual stress state on indentation and hardness testing. Acta Mater., 56, pp. 6205-6213, 2008.

[9] Chen, X., Ogasawara, N., Zhao, M. \& Chiba, N., On the uniqueness of measuring elastoplastic properties from indentation: The indistinguishable mystical materials. J. Mech. Phys. Solids, 55, pp. 1618-1660, 2007.

[10] Chang, W.V. \& Sun, S.C., Nonlinear elastic analysis of the hardness test on rubber-like materials. Rubber Chemistry and Technology, 64, pp. 202210, 1991.

[11] Giannakopoulos, A.E. \& Panagiotopoulos, D.I., Conical indentation of incompressible rubber-like material. Int. J. Solids Struct., 46, pp. 14361447, 2009.

[12] Zisis, Th., Zafiropoulou, V.I. \& Giannakopoulos, A.E., The adhesive contact of a flat punch on a hyperelastic substrate, subject to a pull-out force of a bending moment. Mechanics of Materials, 43, pp. 1-24, 2011.

[13] Lin, Y.Y. \& Chen, H.-Y., Effect of large deformation and material nonlinearity on the JKR (Johnson-Kendall-Roberts) test of soft elastic materials. Journal of Polymer Science B: Polymer Physics, 44, pp. 29122922, 2006.

[14] Green, A.E., Rivlin, R.S. \& Shield, R.T., General theory of small elastic deformations superposed on large elastic deformations. Proc. Royal Society, A21, pp. 128-154, 1952.

[15] Woo, T.C. \& Shield, R.T., Fundamental solutions for small deformations superposed a finite biaxial extension of an elastic body. Arch. Rational Mechanics Analysis, 9, pp. 196-224, 1962.

[16] Dhaliwal, R.S., Singh, B.M. \& Rokne, J.G., Axisymmetric contact and crack problems for an initially stressed neo-Hookean elastic layer. Int. J. Eng. Sci., 18, pp. 169-179, 1980.

[17] Beatty, M.F. \& Usmani, S.A., On the indentation of a highly elastic halfspace, Quart J. of Mech. and Appl. Math., 28, pp. 47-62, 1975.

[18] Karduna, A.R., Halperin, H.R. \& Yin, F.C.P., Experimental and numerical analysis of indentation in finite-sized isotropic and anisotropic rubber-like materials. Annals of Biomedical Engineering, 25, pp. 1009-1016l, 1997.

[19] Humphrey, J.D., Halperin, H.R. \& Yin, F.C.P., Small indentation superimposed on a finite equibiaxial stretch: implications to cardiac mechanics. J. Appl. Mech., 59, pp. 1108-1111, 1991.

[20] Biot, M.A., Mechanics of Incremental Deformations, Wiley, New York, 1965.

[21] Harding, J.W. \& Sneddon, L.N., The elastic stresses produced by the indentation of the plane surface of a semi-infinite elastic solid by a rigid punch. Proc. Cambridge Philos. Soc., 41, pp. 16-26, 1945.

[22] Martinez-Martinez, D. \& De Hosson, J.Th.M., On the deposition and properties of DLC protective coatings on elastomers: A critical review. Surf \& Coat. Tech., 258, pp. 677-690. 